\title{
Contribution à l'étude de l'usure des prothèses totales de hanche. Approche expérimentale sur simulateur
}

\author{
Atef Boulila ${ }^{1, a}$, Khemaïes Jendoubi ${ }^{1}$, Patrick Chabrand ${ }^{2}$, Ali ZGhal ${ }^{3}$ \\ ET Mustapha Khadhraoui ${ }^{4}$ \\ 1 Laboratoire de Biomécanique Orthopédique (LBO), INO. M.T. Kassab, Ksar Saïd, 2010 Manouba, Tunisie \\ 2 Laboratoire LABM UMSR, Université de la Méditerranée, 163 avenue de Luminy, case 918, 13288 Marseille, France \\ 3 Laboratoire de Mécanique des solides, des structures et de développement technologique, ESSTT, BP 56 Beb Mnara, \\ 1008 Tunis, Tunisie \\ 4 Université de Provence/UNIMECA, Technopôle de Château Gombert, 60 rue F. Joliot Curie, \\ 13453 Marseille Cedex 13, France
}

Reçu le 7 novembre 2007, accepté le 20 mai 2008

\begin{abstract}
Résumé - L'évaluation d'un implant, en terme de contrôle qualité, ne peut se faire en définitive que par l'investigation clinique. Cette démarche ne devrait pas toucher seulement les nouveaux implants mais s'appliquer aussi à toute modification même légère d'un implant déjà existant et cliniquement éprouvé. L'investigation clinique prospective reste cependant une approche lourde, contraignante et longue. Elle n'est entreprise actuellement que pour de nouveaux implants ou lorsqu'une modification véritablement innovante est apportée à un implant déjà existant [1]. Dans le cadre du développement d'un nouvel implant articulaire et notamment lors de la conception d'une nouvelle géométrie ou lors du choix d'un nouveau procédé de stérilisation, il pourrait être intéressant de posséder un outil de test pré-clinique permettant de prévoir le comportement et les performances de cette prothèse avant d'investir des moyens lourds dans une étude clinique qui reste cependant indispensable à terme. Les essais tribologiques sur simulateurs offrent cette possibilité [2]. Cette étude porte sur la mise au point et la validation d'un simulateur pour prothèses totales de hanche. À travers nos résultats expérimentaux, développés sur tribomètre à trois unités, nous montrons l'importance des effets du procédé de stérilisation, ainsi que le rôle que peut jouer le milieu biologique et les couples de matériaux de friction (tête et cotyle de la prothèse) sur la tenue en service des prothèses totales de hanche $(\mathrm{PTH})$. Les prothèses de hanche de diamètre plus élevé $(\varnothing 28 \mathrm{~mm})$ et le choix du couple (CoCrMo/polyéthylène) semblent donner un taux d'usure plus faible, et en conséquence, une meilleure longévité des arthroplasties totales de hanche [3].
\end{abstract}

Mots clés : Arthroplastie / usure / bio-tribologie / stérilisation / simulateur

Abstract - Contribution to the study of total hip prostheses wear. Experimental approach on simulator. The evaluation of an implant, in terms of quality control, can be done ultimately only by the clinical investigation. This step should not touch only the new implants but also should be applied to any modification even if it is light of an already existing implant. The prospective clinical investigation remains however a heavy, constraining and long approach. It is undertaken currently only for new implants or when a modification truly innovating is made to an already existing implant [1]. Within the framework of the development of a new articular implant and in particular when in a new designing geometry or during the modification of this one, it could be interesting to have a tool for pre test clinical making it possible to envisage the behaviour and the performances of this prosthesis before investing heavy means of a clinical study which remains however in the long term, essential. The tribological tests on simulators can offer this possibility [2]. In this study, we show the importance of the effects of the sterilization process, as can exploit the role of the biological environment and the materials couple (prosthesis head and cup). The higher hip prostheses diameter $(\varnothing 28 \mathrm{~mm})$ and the choice of the CoCrMo/polyethylene couple seem to give a lower wear rate and consequently a better longevity of the arthroplasty [3].

Key words: Arthroplasty / wear / biotribology / sterilisation / simulator

a Auteur pour correspondance : atef.boulila@laposte.net 

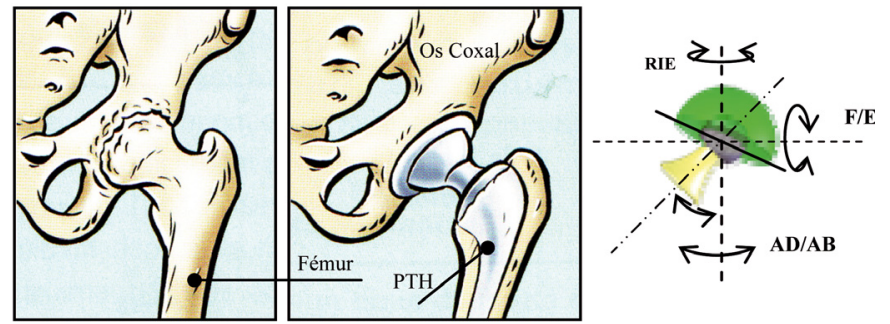

Fig. 1. Articulation coxo-fémorale naturelle (a) et artificielle (b).

\section{Introduction}

La hanche est une articulation synoviale sphéroïde, dont les deux fonctions essentielles sont la transmission des charges physiologiques et la locomotion du corps humain. Elle relie l'os coxal à la tête proximale du fémur. La stabilité du squelette humain dépend en grande partie de la persistance et de la qualité fonctionnelle de cette articulation.

Sur le plan biomécanique, l'articulation de la hanche naturelle chez le bipède constitue une énarthrose qui oriente le membre inférieur dans toutes les directions de l'espace. Cette articulation possède trois axes et trois degrés de liberté [4] : flexion-extension $\left( \pm 23^{\circ}\right)$, adduction-abduction $\left( \pm 6^{\circ}\right)$, rotation externe-rotation interne $\left( \pm 6^{\circ}\right)$. Sur le plan anatomophysiologique, la liaison coxo-fémorale est une biostructure mécaniquement déformable (cartilage) (Fig. 1).

Cette articulation, durant la vie du sujet, peut être sujette à des pathologies ou des traumatismes graves nécessitant de la remplacer par la pose d'un implant articulaire (PTH). On parle alors d'arthroplastie totale de hanche (ATH).

Le marché mondial des prothèses totales de hanche compte actuellement plus de 500 modèles différents, dont au moins 50 sur le marché français, et une dizaine sur le marché tunisien. Tous ces modèles, bien que différents, sont très semblables aussi bien au niveau de la forme et des matériaux utilisés, que de leurs revêtements de surface. Cependant, il a été montré que de faibles différences ou de faibles modifications des caractéristiques d'un implant pouvaient conduire à des différences significatives du comportement clinique à court ou à long terme.

Skuteck et al. [5], montrent que le taux de reprise des PTH augmente régulièrement. Ce taux peut varier de $9,2 \%$ au Canada à $17,5 \%$ aux États-Unis après une période de 13 ans. Selon notre étude rétrospective [3] effectuée à l'Institut National d'Orthopédie M.T. Kassab (Tunisie), plusieurs cas de reprise de PTH ont été enregistrés sur une période de 5 ans (2002 à 2007). Sur les 47 dossiers exploitables, avec un recul suffisant, 45 dossiers présentent des cas de descellement dont la principale cause est l'usure de la composante acétabulaire des PTH implantées.

Sur le plan clinique, la défaillance de ce matériel nécessite l'implantation d'une prothèse de reprise, qui
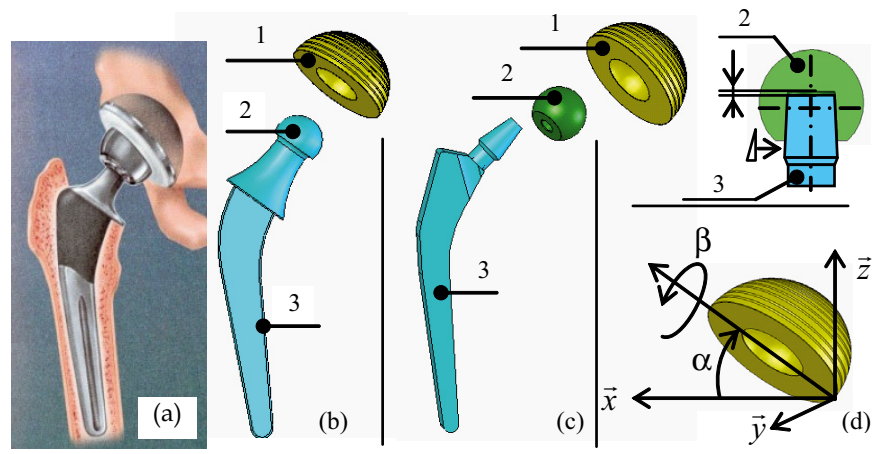

Fig. 2. Arthroplastie totale de hanche (a), solution monobloc (b), solution hybride (c), orientation du cotyle dans les plans anatomiques (d), (1) : cotyle, (2) tête de PTH, (3) tige de PTH.

peut soulever des risques de complication et entraîner des pertes de substances osseuses.

Le recours à l'expérimentation et aux essais mécaniques sur des prothèses articulaires doit permettre de mieux prédire la durée de vie de ces produits. Pour des raisons d'éthique, ces essais doivent être menés en laboratoire, sur des simulateurs de hanche bien adaptés, dans lesquels le milieu biologique et la position géométrique de la PTH doivent être reproduits.

\section{Arthroplastie totale de hanche}

Une prothèse totale de hanche est une articulation artificielle composée principalement de deux composantes : fémorale (ancrée au canal médullaire) et cotyloïdienne implantée à l'os coxal du patient. Il existe plusieurs types d'implants articulaires de hanche à couples de matériaux différents et de géométries et de tailles très variées.

\subsection{PTH monoblocs et hybrides}

Plusieurs facteurs d'ordre pathologique (état de l'os) ou socioéconomique (coût, conditions du patient...), peuvent guider le praticien dans le choix de la PTH à implanter (hybride, avec céramique,...). Actuellement les choix de modèles de PTH et des couples de matériaux de frottement dépendent de l'orientation académique des écoles d'orthopédie, mais restent arbitraires dans la majorité des cas.

Le compartiment fémoral peut être monobloc (PTH type Charnley) (Fig. 2b) ou hybride (tête + corps à col conique) (PTH type Müller) (Fig. 2c). Selon l'état de l'os et l'âge du patient, le protocole opératoire peut opter pour une implantation avec ou sans ciment. Dans le cas de la solution sans ciment, un traitement de surface de la tige de la PTH par hydroxyapatite, favorisant un ancrage biologique de l'implant dans l'os peut être utilisé.

Pour le compartiment cotyloïdien, la technique chirurgicale doit respecter deux angles caractéristiques d'inclinaison, formé par le plan horizontal et le plan de la cupule 
Tableau 1. Principaux couples de matériaux utilisés en arthroplastie totale de hanche.

\begin{tabular}{|l|c|c|c|}
\hline Matériaux prothétiques & Cotyle & $\begin{array}{c}\text { Tête } \\
\text { de PTH }\end{array}$ & $\begin{array}{c}\text { Corps } \\
\text { de PTH }\end{array}$ \\
\hline UHMWPE & $\checkmark$ & & \\
\hline Acier inoxydable 316L & $\checkmark$ & $\checkmark$ & $\checkmark$ \\
\hline CoCrMo & & $\checkmark$ & $\checkmark$ \\
\hline $\begin{array}{l}\text { Alumine }\left(\mathrm{Al}_{2} \mathrm{O}_{3}\right) \\
\text { et zircone }\left(\mathrm{ZrO}_{2}\right)\end{array}$ & $\checkmark$ & $\checkmark$ & \\
\hline Titane & & & $\checkmark$ \\
\hline
\end{tabular}

$(\alpha)$, et d'antéversion, $(\beta)$ autour de $\boldsymbol{u}$, comme le montre la figure $2 \mathrm{~d}$.

\subsection{Matériaux prothétiques}

Plusieurs couples de matériaux de friction sont utilisés dans l'arthroplastie totale de hanche. Ils doivent répondre à des critères de biocompatibilité, de biofonctionnalité mais aussi, de résistance à la fatigue et à la corrosion pour favoriser de meilleures performances mécaniques et tribologiques. Les biomatériaux couramment utilisés en chirurgie orthopédique sont regroupés en trois familles : les matériaux métalliques (acier inoxydable, $\mathrm{Ti}_{6} \mathrm{Al}_{4} \mathrm{~V}, \mathrm{CoCrMo}$ ), les matières thermoplastiques (UHMWPE, ciment acrylique) et les céramiques (alumine, zircone) (Tab. 1). D'autres matériaux plus évolués (composite à fibre de carbone,...) sont à l'étude pour des validations cliniques.

En chirurgie orthopédique, la majorité des prothèses articulaires (hanche, genou, épaule,...) font intervenir le polyéthylène à très haute densité (UHMWPE), pour sa biocompatbilité, son effet amortissant (damping) et son coût relativement bas.

Toutefois, sous l'action des sollicitations cycliques et de l'environnement, l'usure du polyéthylène (adhésive, abrasive ou par fatigue) se traduit par des endommagements qui sont encore mal expliqués : déformations visco-plastiques par écoulement (ou fluage), enlèvement de matière, délaminage, fissuration, etc. (Fig. 3). Plusieurs travaux montrent que des usures de plusieurs millimètres peuvent se produire au bout de quelques années et qu'il convient d'améliorer le produit et ses conditions de fonctionnement pour en limiter les déformations.

Quant aux céramiques, plusieurs travaux montrent qu'elles ont une bonne tenue à l'usure et sont utilisées aussi bien dans la fabrication des cotyles que les têtes de PTH.

Les matériaux à base de titane, sont utilisés dans la fabrication des tiges de PTH en vue de favoriser un meilleur ancrage à l'os. La faible densité et le module d'élasticité réduit de cet alliage comparé à l'acier inoxydable 316L, font que ce matériau est mieux toléré par le tissu osseux.
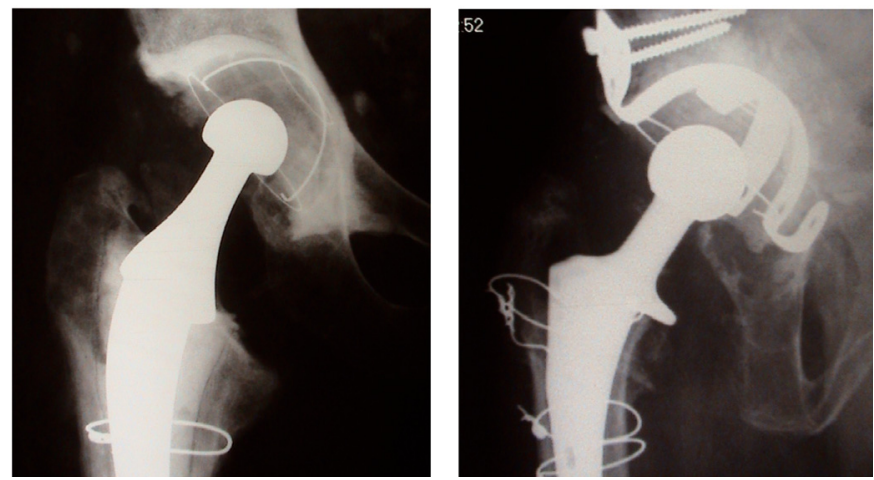

Fig. 3. Cliché radiologique de cotyle enfoncé (cas traités à l'Institut M.T. Kassab).

\section{3 Échecs arthroplastiques de la hanche}

Les mécanismes prédominants du descellement des prothèses articulaires étaient considérés pendant longtemps comme des paramètres mécaniques, liés par exemple aux forces de frottement excessives entre les pièces articulaires, ou aux propriétés mécaniques des matériaux prothétiques (ciment...).

Depuis quelques années, plusieurs auteurs [6,7] ont mis l'accent sur la prédominance des facteurs biologiques, et notamment la réaction aux particules dues à l'usure des surfaces articulaires, ou à la mobilité des implants : ainsi se sont développées d'autres recherches, s'orientant préférentiellement vers la mise au point de couple de matériaux donnant naissance à moins de débris, ou vers une stabilité prothétique primaire accrue. De nouveaux concepts sont proposés pour améliorer l'interface périprothétique (os/prothèse) dont la défaillance est liée à la micromobilité de l'implant, mais surtout à la présence de débris d'usure de la prothèse entraînant une lyse osseuse ou l'apparition d'une interface fibreuse.

L'observation clinique (radiologie, histologie...) montre que la plus grande majorité des débris d'usure retouvés autour d'une prothèse articulaire fonctionnelle sont produits par le couple tête/cupule. Sur le plan radiologique, on note en effet les relations étroites entre le descellement cotylö̈dien et la pénétration de la tête prothétique dans la cupule. Ainsi Postel et al. [8] sur 476 prothèses de Charnley revues au-delà de 10 ans, trouvent que $11,3 \%$ de l'usure de la cupule est supérieure à $2 \mathrm{~mm}$ dans l'ensemble de la série. Mais s'il ne considère que les prothèses présentant un liseré cotylö̈dien, de plus de $2 \mathrm{~mm}$ d'épaisseur, $31 \%$ de ces prothèses comportent une usure de plus de $2 \mathrm{~mm}$. De même, une autre série [8] comparant 50 prothèses de Müller et 50 prothèses de Charnley à plus de 10 ans, montre cette relation : parmi les 50 prothèses de Charnley 46 prothèses présentent une usure de moins de $2 \mathrm{~mm}$ et 45 cupules sont sans liseré. À l'inverse, parmi les 50 prothèses de Müller, il n'y a que 21 usures inférieures à $2 \mathrm{~mm}$ et 27 cupules sans liseré. 


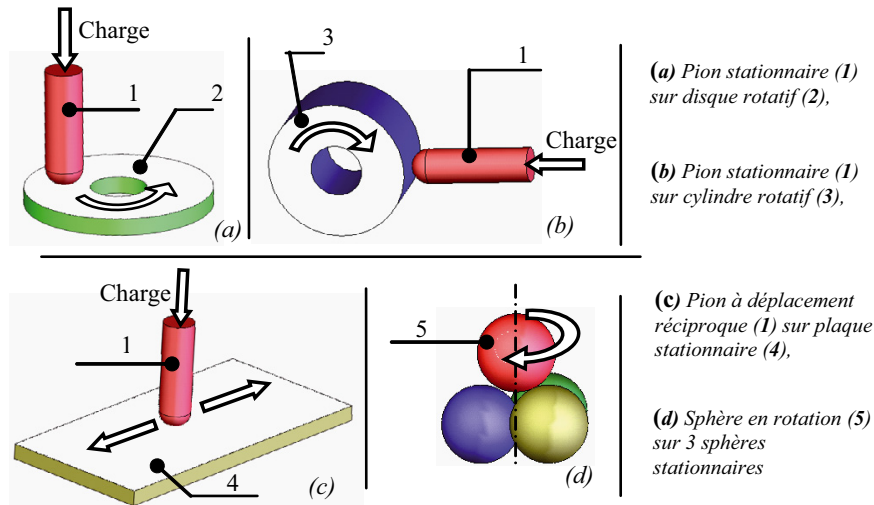

Fig. 4. Essais universels d'usure.

\section{Tribologie et essais d'usure}

La tribologie qui est la science des frottements des surfaces en contact animées d'un mouvement relatif, entre en jeu de façon significative dans de nombreux domaines industriels, mais intervient également dans les systèmes vivants naturels ou prothétiques [9]. L'usure est considérée comme étant la conséquence d'un mauvais agencement du contact entre les pièces de friction.

Dans le domaine de la biomécanique ostéo-articulaire, l'évaluation de l'usure in vitro fait appel à trois techniques de mesures : essais universels, essais sur machines asservies et essais sur simulateurs. Tous ces essais peuvent être menés à sec ou dans un milieu à lubrification contrôlée.

\subsection{Essais universels d'usure}

Ces essais conduits généralement sur des éprouvettes, utilisent des concepts géométriques simples. Les interfaces de contact sont caractéristiques des tests de frottement et d'usure au glissement ou au roulement. Les essais sont effectués grâce à des appareillages définis par les schémas de la figure 4 [10].

\subsection{Essais d'usure sur machines asservies}

Ces essais sont conduits sur prothèses en milieu contrôlé. L'implant est fixé à la machine d'essais [11,12]. Et l'intégration d'un mécanisme de transmission du chargement permet de reproduire les mouvements imposés à l'articulation prothétique (Fig. 5).

\subsection{Essais d'usure sur simulateurs - État de l'art}

Les simulateurs (ou tribomètres) pour implants articulaires existent sous différentes formes et sont utilisés en biomécanique pour plusieurs raisons : économique, de fiabilité, mais aussi de disponibilité. Affatato et al. [13] Saikko et al. [15] et Essner et al. [14] sont parmi les auteurs qui ont justifié le choix de cet outil expérimental pour surmonter certaines contraintes expérimentales
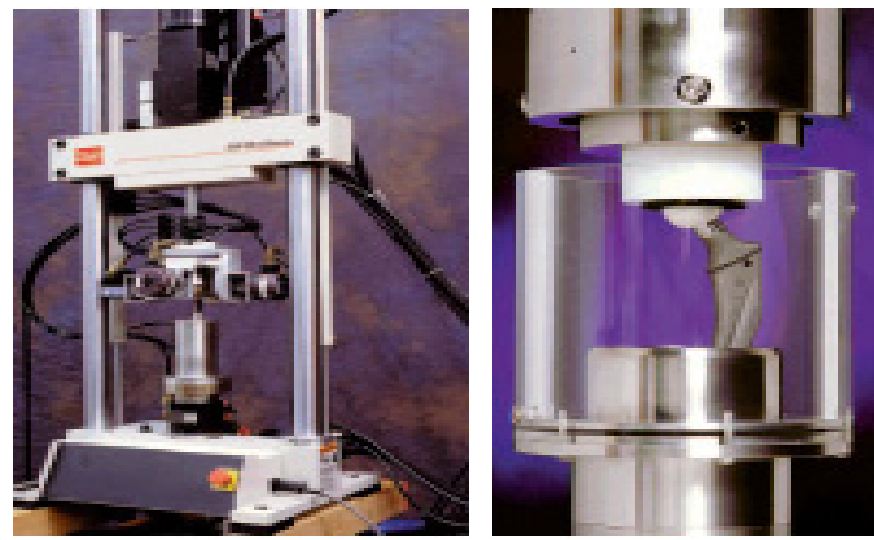

Fig. 5. Essais d'usure sur machines asservies (cas de la machine MTS).

(géométrique, cinématique...) et suivre des protocoles d'essais plus adaptés à l'implant.

Pour la majorité des auteurs [12-15], les tribomètres de hanche permettent d'obtenir des résultats expérimentaux plus fidèles à la réalité que les autres dispositifs. En effet, la cinématique articulaire (3 degrés de liberté), les conditions de chargement et le milieu biologique, sont des paramètres contrôlables sur simulateur qui peuvent altérer les résultats expérimentaux dans la plupart des cas. Bien que ces appareils ne puissent fournir que des résultats pré-cliniques, ils ont le mérite de donner différentes indications sur la performance des prothèses articulaires à implanter et la réponse mécanique des biomatériaux utilisés.

\section{Matériels et méthodes}

Cette étude est justifiée par le nombre élevé de cotyles endommagés et prélevés sur des sujets traités à l'Institut National d'Orthopédie M.T. Kassab (Tunisie). Le travail porte dans un premier temps sur des analyses métrologiques par scanner 3D de 47 cotyles explantés et fabriqués en polyéthylène haute densité. La deuxième étape de l'étude porte sur la conception et l'exploitation d'un simulateur, pour conduire des essais d'usure de PTH, type Müller (hybride) dans un environnement contrôlé.

\subsection{Mesure de l'enfoncement sur cotyles explantés}

Pour localiser le déplacement et la direction principale de l'usure des cotyles sélectionnés, nous avons procédé à une acquisition tridimensionnelle des morphologies de surface à l'aide d'une machine type PICZA30 Roland ${ }^{\circledR}$ de précision 5/100 de $\mathrm{mm}$. Un palpeur mécanique permet l'acquisition d'un nuage de points matérialisant les états sain et usé de la cupule (Fig. 6). À l'aide d'un logiciel de conception $\mathrm{CAO}$, nous analysons les deux surfaces scannées et déterminons le volume de chaque composant. Le volume usé est donné par $\Delta V=V_{2}-V_{1}\left(\right.$ en $\left.\mathrm{mm}^{3}\right)$, 

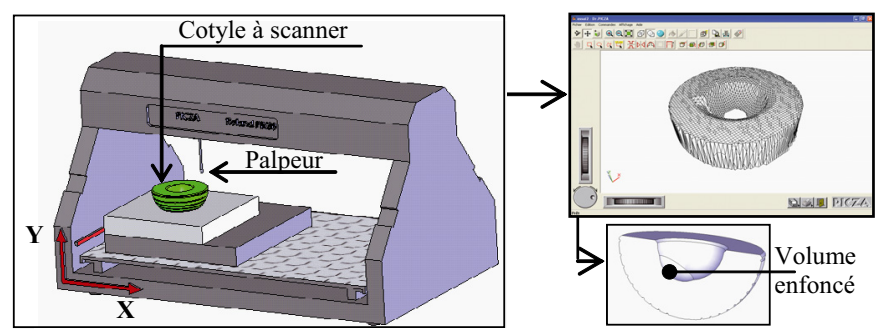

Fig. 6. Scanner $3 \mathrm{D}$ type PICZA 30 Roland $^{\circledR}$ (a), cupule scannée (b).

Tableau 2. Usure volumétrique des PTH par rétro conception - cas du couple polyéthylène/acier inoxydable.

\begin{tabular}{|c|c|c|}
\hline & $\varnothing=22,2 \mathrm{~mm}$ & $\varnothing=28 \mathrm{~mm}$ \\
\hline$V_{1}\left(\mathrm{~mm}^{3}\right)$ & 1182,24 & 5747,02 \\
\hline$V_{2}\left(\mathrm{~mm}^{3}\right)$ & 1254,97 & 5831,18 \\
\hline$\Delta V\left(\mathrm{~mm}^{3}\right)$ & $\mathbf{7 2 , 7 3 8}$ & $\mathbf{1 3 4 , 9 1 4}$ \\
\hline
\end{tabular}

où $V_{1}$ et $V_{2}$ représentent respectivement les volumes de la surface interne du cotyle explantée et neuve.

La moyenne des résultats les plus significatifs sur une série de 47 prothèses analysées $(\varnothing=22,2 \mathrm{~mm}$ et $\varnothing=$ $28 \mathrm{~mm}$ ) sont regroupés dans le tableau 2. L'âge moyen de l'implantation avant reprise de PTH est de 8,24 ans.

Sur le plan tribologique, l'usure de la cupule (polyéthylène) in vivo, entraîne la création d'un jeu entre la tête et le cotyle de la prothèse. La persistence des forces répétitives appliquées sur la tête lors de la marche, donne naissance à des déformations conduisant à des compressions de volume (enfoncement de plusieurs $\mathrm{mm}^{3}$ ).

\subsection{Matériaux de friction et types de prothèses testées}

Tous les essais d'usure sont conduits sur des prothèses neuves (tirées de l'emballage) type Müller à têtes rapportées. Les dimensions des prothèses testées sont choisies en fonction des modèles standards couramment utilisés à l'Institut M.T. Kassab [17]. Quatre couples de matériaux de friction sont sélectionnés pour l'étude, mais notre intérêt a porté sur le couple (polyéthylène/acier inoxydable) utilisé fréquemment dans la majorité des centres orthopédiques (Tab. 3).

\subsection{Caractéristiques du simulateur utilisé (TUNIS-3DOF)}

Le simulateur utilisé TUNIS-3DOF (TUNisian Hip Simulator - 3 Degrees Of Freedom), est conçu en s'inspirant des modèles existants dans la littérature [13-15]. Il est composé de quatre unités : la commande, le chargement, le mouvement et les essais (Fig. 7, Tab. 4)

Des options sont ajoutées pour optimiser le temps des essais et explorer plusieurs paramètres physico-chimiques pouvant affecter les implants articulaires. Le simulateur comporte trois unités disposées à $120^{\circ}$ pour accueillir
Tableau 3. Dimensions des cotyles utilisés.

\begin{tabular}{|c|c|c|c|c|c|c|c|c|}
\hline $\boldsymbol{d} / \boldsymbol{D}$ & \multicolumn{10}{|c|}{$D(\mathrm{~mm})$} \\
\hline $\boldsymbol{d}(\mathrm{mm})$ & 48 & 50 & 52 & 54 & $\mathbf{5 6}$ & 58 & 60 & 62 \\
\hline 22,2 & 0,46 & 0,44 & 0,42 & 0,41 & 0,39 & 0,38 & 0,37 & 0,35 \\
\hline $\mathbf{2 8}$ & 0,58 & 0,56 & 0,53 & 0,51 & $\mathbf{0 , 5 0}$ & 0,48 & 0,46 & 0,45 \\
\hline 32 & 0,66 & 0,64 & 0,61 & 0,59 & 0,57 & 0,55 & 0,53 & 0,51 \\
\hline 36 & 0,75 & 0,72 & 0,69 & 0,66 & 0,64 & 0,62 & 0,60 & 0,58 \\
\hline 40 & 0,83 & 0,80 & 0,76 & 0,74 & 0,71 & 0,68 & 0,66 & 0,64 \\
\hline
\end{tabular}
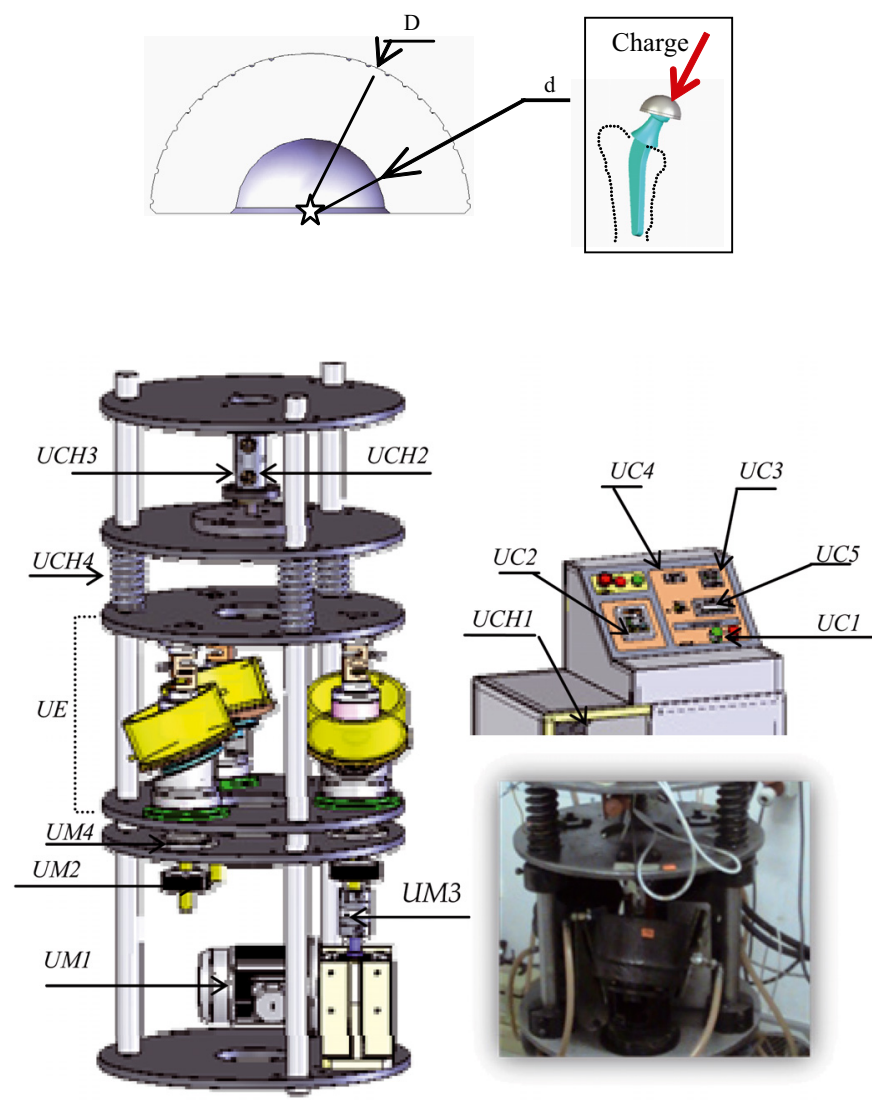

Fig. 7. Simulateur de Hanche TUNIS-3DOF.

les prothèses à tester, soit à l'air libre ou dans un milieu contrôlé (sérum, solution Ringer,...). Ces unités sont équipées d'un système de régulation de température permettant de contrôler la température d'essais $\left(37^{\circ} \mathrm{C}\right)$.

Un variateur électronique permet de sélectionner la fréquence d'essai $(0,1$ à $2,5 \mathrm{~Hz})$. Le chargement de la prothèse est fait en utilisant un système hydromécanique (ressorts + vérin) permettant d'imposer une charge proche de la sollicitation physiologique, et varie de 100 à $6000 \mathrm{~N}$. Le dispositif à ressorts permet de garder un contact permanent entre les pièces prothétiques et éviter les problèmes de décoaptation de la tête de la prothèse par rapport au cotyle, phénomène qui peut être rencontré in vivo. Le contrôle de la charge est assuré par des capteurs à jauges, interposés à $120^{\circ}$ par rapport à l'axe du tribomètre pour fournir la sollicitation imposée à la prothèse à l'aide d'un étalonnage préétabli. 
Tableau 4. Nomenclature - simulateur TUNIS-3DOF.

\begin{tabular}{|c|l|l|}
\hline Unité & \multicolumn{2}{|c|}{ Composants } \\
\hline $\begin{array}{c}\text { Unité de } \\
\text { commande : } \\
\text { UC }\end{array}$ & $\begin{array}{l}\text { UC1 - Boutons M/A, AU }- \text { Variateur de fréquence } \\
\text { UC3 - Régulateur de température }\end{array}$ & $\begin{array}{l}\text { UC-4 - Compteur de cycles } \\
\text { UC-5 - Acquisition de chargement }\end{array}$ \\
\hline Unité de \\
chargement : & UCH1 - Pompe hydraulique & UCH3 - Vérin \\
UCH & UCH2 - Flexibles & UCH3 - Ressorts \\
\hline Unité de & & UM3 - Joint d'Oldham \\
mouvement : & UM1 - Moto-réducteur & UM4 - Kits de roulement \\
UM & UM2 - Roues et courroie crantée & UE4 - Échangeur thermique \\
\hline Unité & UE1 - Modules d'essais & UE5 - Tige anti-rotation et lumière \\
d'essais : & UE2 - Éprouvettes d'essais & \\
UE & UE3 - Roulement double appuis &
\end{tabular}

Tableau 5. Principales configurations de module d'essais existants dans la littérature.

\begin{tabular}{|c|c|c|c|c|c|c|c|c|}
\hline & \multicolumn{4}{|c|}{ Disposition 1 } & \multicolumn{4}{c|}{ Disposition 2 } \\
\hline Cotyle & & $\mathrm{MO}$ & $\mathrm{CH}$ & $\mathrm{MO}$ & $\mathrm{CH}$ & & & \\
\hline Tête de PTH & $\mathrm{CH}$ & & & & & $\mathrm{MO}$ & $\mathrm{CH}$ & $\mathrm{MO}$ \\
\hline Module d'essais & Configuration 1 & \multicolumn{1}{|c|}{ Configuration 2 } & \multicolumn{1}{c|}{ Configuration 3 } & Configuration 4 \\
\hline
\end{tabular}

$\mathrm{CH}$ : chargement, $\mathrm{MO}$ : mobilité.
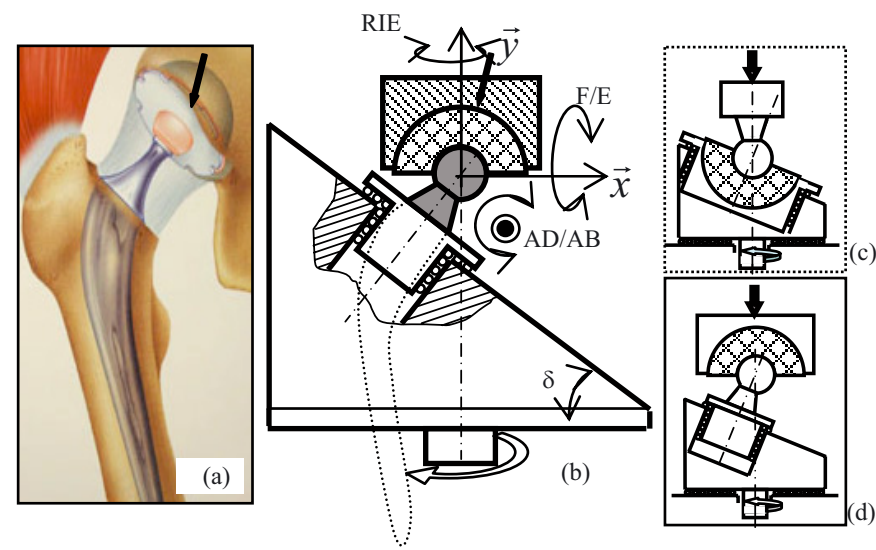

Fig. 8. Dispositions des modules d'essais : position anatomique de la PTH in vivo (a), position de la PTH in vitro (b), disposition 1 (c), disposition 2 (d).

\subsubsection{Unité et modules d'essais}

Le principal module dans l'architecture du simulateur est le mécanisme d'amarrage et de fixation de la prothèse à tester, par ses deux composantes fémorale (tête) et acétabulaire (cotyle). L'éprouvette est immergée dans une enceinte comportant un liquide reproduisant le milieu biologique (Figs. 8a et b). Des travaux de la littérature [13-15] montrent qu'il existe deux dispositions pour le maintien des PTH sur simulateur :

- Disposition 1 : cotyle au-dessus de la tête de la prothèse, conformément à la disposition anatomique de la prothèse (Fig. 8d).
- Disposition 2 : cotyle en dessous de la tête (Fig. 8c).

Les travaux de Galvin et al. [18], montrent que les résultats des essais ne sont pas affectés par ces deux types de dispositions (1 ou 2) qui peuvent donner des résultats identiques sous les mêmes conditions (chargement et mouvement) pour un même couple de matériaux. Le tableau 5 regroupe les principales configurations rencontrées chez Affatato et al. [13], Saikko et al. [15], Jin et al. [16] et Essner et al. [14]. La disposition 2 est la solution technologique retenue pour la conception et la réalisation du tribomètre TUNIS-3DOF.

La mobilité de la prothèse est assurée par l'unité de mouvement composée d'un variateur et d'un motoréducteur. Le chargement de la prothèse est réalisé par un système composé de vérin et de ressorts. La multiplication des modules et la planification des manipulations par la méthode des plans d'expériences de Taguchi [19] a permis de mieux optimiser le temps d'essais. En effet, pour planifier le protocole expérimental, nous avons montré que le temps de réalisation d'un million de cycles, pour une fréquence de $1 \mathrm{~Hz}$, dépasse les 12 jours avec une durée moyenne de fonctionnement de 20 heures sur 24. Ceci met en question le temps total alloué à l'ensemble des essais pour les différents couples de matériaux, et justifie notre choix d'opter pour un simulateur à trois unités.

\subsubsection{Contrôle et acquisition des données}

Dans sa version actuelle, le contrôle et l'acquisition des données expérimentales sur le simulateur TUNIS-3DOF est assuré par un ensemble de composants 


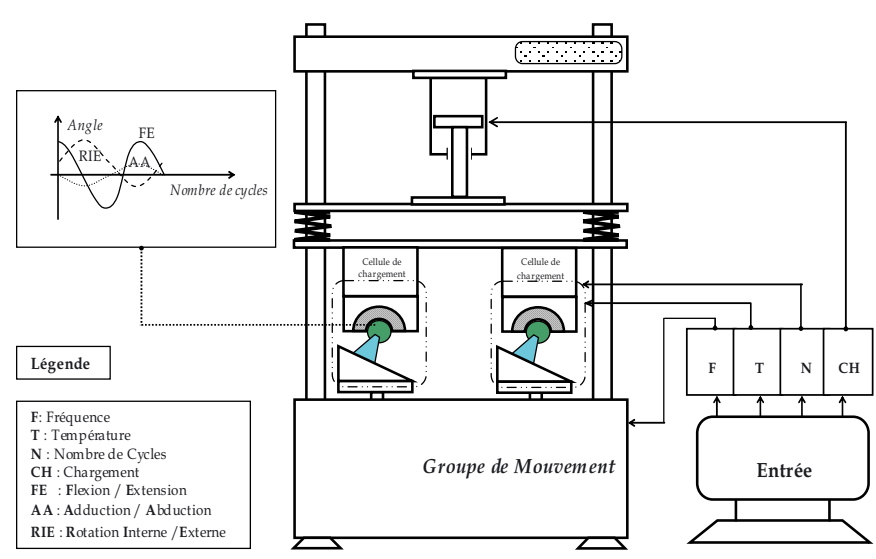

Fig. 9. Acquisition des données sur le simulateur de hanche.

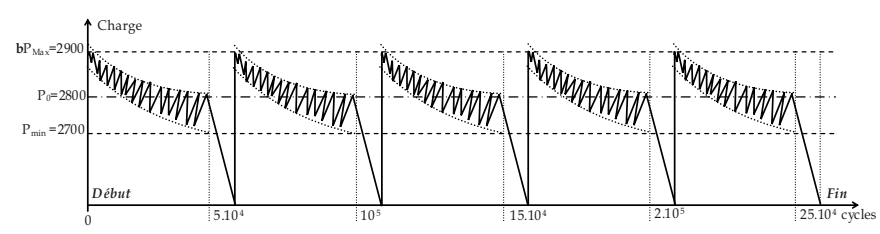

Fig. 10. Spectre de chargement.

mécaniques, hydrauliques et électroniques, composé principalement d'un variateur de fréquence, d'un régulateur de température, d'un compteur de cycles et d'un système de mise en charge (Fig. 9).

\subsubsection{Unité de mise en charge}

La charge moyenne $\left(P_{0}\right)$ appliquée aux trois prothèses (disposées à $120^{\circ}$ ) est générée par un système hydromécanique (pompe + vérin + ressorts) et contrôlée par des capteurs de forces interposés dans l'axe de chargement des PTH. Selon le protocole d'essai prédéfini (fréquence d'essai et temps d'arrêt pour mesure), le spectre de chargement appliqué à la prothèse du début jusqu'à la fin de l'essai, prend une allure oscillante par rapport à la charge moyenne $P_{0}$ (Fig. 10).

En admettant que la charge physiologique appliquée à la hanche, lors de la marche, diffère d'un sujet à un autre, la procédure de mise en charge intégrée au simulateur impose aux éprouvettes des cycles de chargement combinés fatigue fluage. Ceci peut s'approcher en partie du chargement observé sur des prothèses implantées sur une longue période de fonctionnement (repos, activité,...).

\subsubsection{Cellules thermostatées}

Pour s'approcher au mieux du milieu physiologique, des cellules thermostatées sont interposées aux trois modules d'essai, pour assurer une convection thermique et imposer au liquide de Ringer une température moyenne d'essai de $37{ }^{\circ} \mathrm{C}$. La cellule, isolée thermiquement, fonctionne grâce à une circulation d'eau chaude par minipompe, contrôlée par un régulateur (Fig. 11).
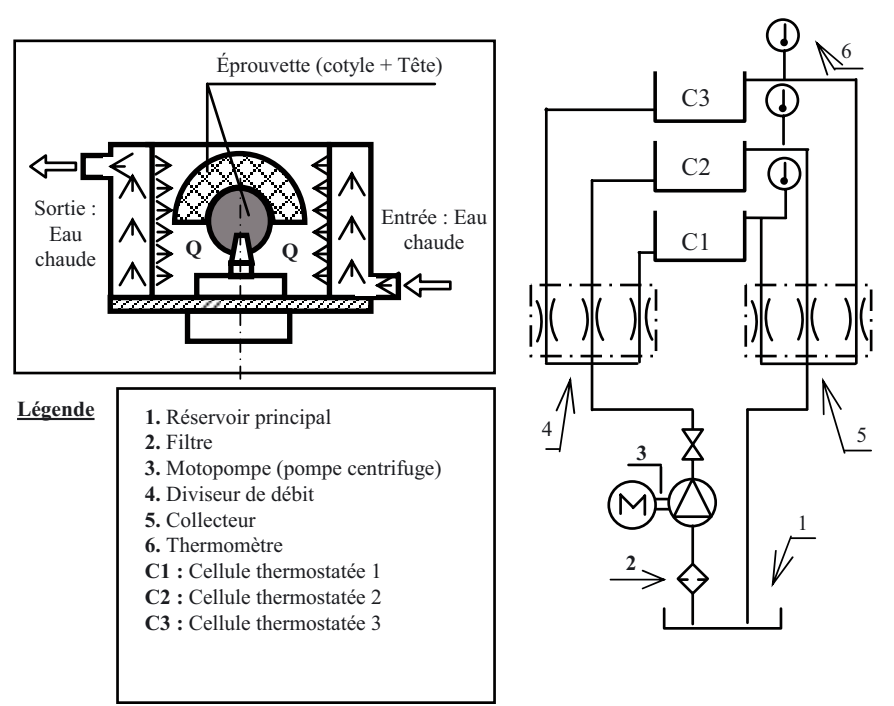

Fig. 11. Cellules thermostatées (a), circuit de distribution de l'eau chaude (b).

\subsection{Protocole expérimental}

Dans cette étude nous procédons à l'exploitation et à la validation du simulateur réalisé. Nous cherchons à établir un modèle empirique de prédiction de la durée de vie en service d'une prothèse totale de hanche de type Müller, en prenant en compte les effets du milieu biologique, la géométrie de la prothèse, les couples des matériaux et la température.

Le protocole expérimental est développé pour conduire des essais tribologiques en tenant compte des paramètres influençant le comportement mécanique (usure) des PTH à têtes rapportées (Fig. 12).

Des travaux dans la littérature [20], montrent l'existence d'une forte sensibilité des cupules acétabulaires à l'usure et à l'environnement. La solution plasmatique de Ringer permet de simuler le milieu biologique lorsqu'elle est utilisée à la température du corps humain $\left(37^{\circ} \mathrm{C}\right)$ avec un $\mathrm{pH}$ compris entre 5,4 et 5,7. Selon Geringer et al. [21], la composition chimique de cette solution est définie par le tableau 6.

D'autres essais utilisant un sérum humain sont programmés en vue d'analyser les effets de certains antibiotiques sur la durée de vie des implants.

\section{Résultats expérimentaux et interprétation des mesures}

\subsection{Conditions des essais et validation du système}

Plusieurs paramètres de nature mécanique ou biologique peuvent gouverner les réactions tribologiques sur prothèses totales de hanche. Notre étude expérimentale, appliquée selon le protocole adopté, porte sur l'évaluation de la perte de masse par analyse du composant cotyloïdien de la prothèse en fonction du nombre de cycles appliqués. 


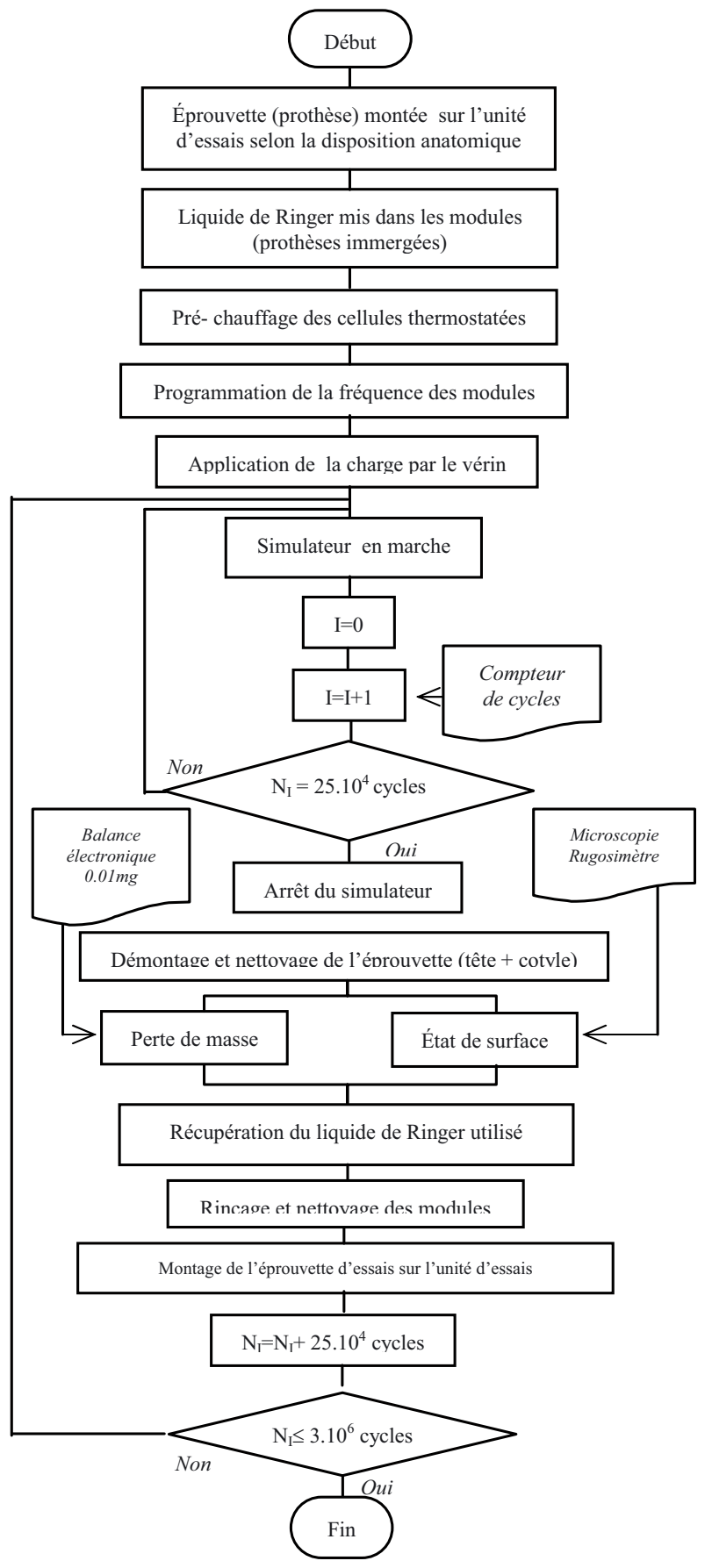

Fig. 12. Protocole expérimental adopté.

En tenant compte de la capacité statique et dynamique de chargement offerte par le simulateur, nous nous sommes limités à une charge maximale de $2900 \mathrm{~N}$ et un nombre de cycles appliqués égal à $3,5 \times 10^{5}$ cycles pour chaque série d'essais. Le nombre total de cycles pouvait cependant dépasser les $5 \times 10^{5}$ cycles pour les couples céramique/céramique. Les caractéristiques des éprouvettes utilisées dans cette étude sont regroupées dans le tableau 7.
Tableau 6. Composition chimique de la solution plasmatique de Ringer.

\begin{tabular}{|c|c|c|c|c|}
\hline Espèce chimique & $\mathrm{NaCl}$ & $\mathrm{KCl}$ & $\mathrm{NaHCO} 3$ & $\mathrm{CaCl}_{2}-2 \mathrm{H}_{2} \mathrm{O}$ \\
\hline $\begin{array}{c}\text { Concentration } \\
\left(\mathrm{g} . \mathrm{l}^{-1}\right)\end{array}$ & 8,5 & 0,25 & 0,2 & 0,3 \\
\hline
\end{tabular}

Tableau 7. Caractéristiques des éprouvettes testées et conditions d'essais.

\begin{tabular}{|c|c|c|c|}
\hline Matériaux & $\begin{array}{c}\text { Module M1 } \\
\text { HD/acier } \\
\text { inoxydable } \\
316 \mathrm{~L}\end{array}$ & $\begin{array}{c}\text { Module M2 } \\
\text { HD/acier } \\
\text { inoxydable } \\
316 \mathrm{~L}\end{array}$ & $\begin{array}{c}\text { Module M3 } \\
\text { HD/acier } \\
\text { inoxydable } \\
316 \mathrm{~L}\end{array}$ \\
\hline $\begin{array}{c}\text { Géométrie } \\
\text { de la cupule } \\
(\mathrm{mm})\end{array}$ & $\varnothing 28 \times 56$ & $\varnothing 28 \times 56$ & $\varnothing 28 \times 56$ \\
\hline $\begin{array}{c}\text { Procédé de } \\
\text { stérilisation }\end{array}$ & Rayon $\gamma$ & Rayon $\gamma$ & $\begin{array}{c}\text { Autoclave } \\
\text { (bloc opératoire) }\end{array}$ \\
\hline $\begin{array}{c}\mathrm{pH} \text { de la } \\
\text { solution }\end{array}$ & 5,7 & 5,4 & 5,4 \\
\hline
\end{tabular}

Plusieurs essais réalisés à l'air libre et sous environnement contrôlé, sont conduits pour assurer le rodage du système et comparer certains résultats de mesure avec ceux déjà existants dans la littérature [13-15]. La courbe relative à la perte de masse pour des conditions d'essais proches de celles adoptées par les autres laboratoires [13-15] semble montrer une même tendance (Fig. 13).

En effet, la majorité des résultats enregistrés sur le tribomètre TUNIS-3DOF donnent des évaluations linéaires de la perte de masse en fonction du nombre de cycles appliqués. Nous constatons que la majorité des diagrammes $(\Delta m, N)$ se compose de deux ou trois stades d'usures [10] :

- Stade 1 : correspond à une évolution rapide de l'usure, caractéristique d'un rodage des surfaces, à une réduction des aspérités et une augmentation de l'aire effective de contact entre la tête de la prothèse et le cotyle.

- Stade 2 : la phase la plus longue, durant laquelle l'augmentation de l'usure reste faible surtout en présence d'un fluide.

- Stade 3 : c'est une phase qui correspond, théoriquement, à l'érosion rapide des couches sous-jacentes du matériau, dont les caractéristiques mécaniques vont en diminuant au fur et à mesure que l'on pénètre dans la matière. Cependant, pour les implants articulaires de hanche, cette phase d'accélération du phénomène d'usure n'est observée qu'après quelques dizaines de millions de cycles et rares sont les cas cliniques qui atteignent ce stade. En effet, l'usure accélérée est aggravée par l'ostéolyse aux interfaces os-prothèse, puis l'activation des mécanismes du problème de 
Tableau 8. Comparaison des résultats d'essais tribologiques.

\begin{tabular}{|c|c|c|c|c|c|c|}
\hline \multirow[t]{2}{*}{ Simulateur } & \multicolumn{4}{|c|}{ Conditions d'essais } & \multicolumn{2}{|c|}{ Usure $/ 10^{6}$ cycles } \\
\hline & Charge $(\mathrm{KN})$ & Fréquence $(\mathrm{Hz})$ & Milieu & Température $\left({ }^{\circ} \mathrm{C}\right)$ & $\Delta m(\mathrm{mg})$ & $\Delta V\left(\mathrm{~mm}^{3}\right)$ \\
\hline HUT-3 [15] & 2,41 & 1,17 & Sérum bovin & $36,3 \pm 0,1$ & $56,4 \pm 13,3$ & $60,36 \pm 14,6$ \\
\hline $\begin{array}{c}\text { MTS, Eden Prairie, } \\
\text { MN }[22]\end{array}$ & 2,45 & 1 & Sérum bovin & - & $5,787-111,265$ & $6,19-119$ \\
\hline \multirow[t]{2}{*}{$\begin{array}{c}\text { Shore Western, } \\
\text { USA [24] }\end{array}$} & 2,0 & 1 & \multirow[t]{2}{*}{ Sérum bovin } & - & $76 \pm 2($ ETO $)$ & $81,32 \pm 2,18$ \\
\hline & 2,0 & 1 & & - & $63 \pm 8$ (Gamma) & $67,42 \pm 8,6$ \\
\hline TUĖIS-3DOF & 2,8 & 1,26 & Ringer & 37,07 & $69,3 \pm 16$ & $74,16 \pm 17,6$ \\
\hline
\end{tabular}

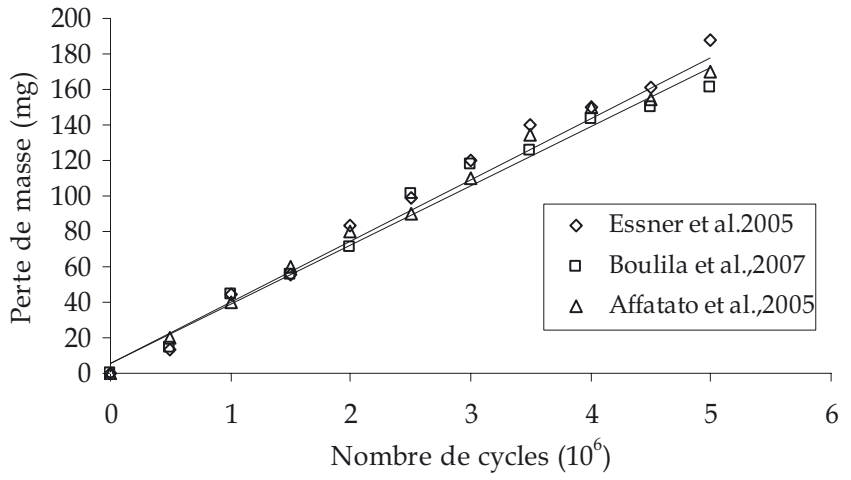

\begin{tabular}{|c|c|c|}
\hline Auteurs & Équation & $\begin{array}{c}\text { Coefficient de } \\
\text { détermination } R^{2}\end{array}$ \\
\hline $\begin{array}{c}\text { Essner } \\
\text { et al. [23] }\end{array}$ & $\Delta m=37,334 . N+2,4668$ & 0,9916 \\
\hline $\begin{array}{c}\text { Présente } \\
\text { étude }\end{array}$ & $\Delta m=33,301 . N+6,0673$ & 0,9803 \\
\hline $\begin{array}{c}\text { Affatato } \\
\text { et al. }[24]\end{array}$ & $\Delta m=34,545 . N+5,4545$ & 0,9911 \\
\hline
\end{tabular}

Fig. 13. Courbe de la perte de masse en fonction du nombre de cycles.

descellement aseptique de l'implant. Les douleurs apparaissent et l'intervention se fait avant l'arrivée au stade d'usure accélérée.

Selon Kapsa et al. [25], certains résultats d'essais tribologiques sont altérés par le type et la nature du tribomètre employé, outre les conditions expérimentales choisies. Des travaux publiés récemment montrent que les taux d'usure gravimétriques (ou volumétriques) des cupules acétabulaires en polyéthylène dépendent de la nature du polymère employé (standard, réticulé,...). Les résultats les plus significatifs sont regroupés dans le tableau 8.

Les résultats obtenus selon notre protocole d'essais, pour le couple (CrCoMo/UHMWPE), sont très proches de ceux présentés par Saikko et al. [15], Essner et al. [23] et Affatato et al. [24] Ces résultats offrent des indications positives sur la validation du simulateur TUNIS-3DOF pour les conditions expérimentales préconisées.

\subsection{Effet du milieu biologique}

Pour s'approcher au mieux des conditions du milieu biologique (in vivo), une solution Ringer est élaborée pour jouer le rôle du liquide synovial. La solution choisie est caractérisée par sa composition chimique (Tab. 7) et un $\mathrm{pH}$ évalué à 5,7 . Un pH inférieur à ce seuil correspond à un milieu plus acide, donc plus agressif. Dans la littérature clinique [26], l'acidité peut se traduire par la présence d'un pourcentage d'éléments alcoolisés dans l'organisme humain.

Outre les problèmes de nécroses qui peuvent affecter l'os, il a été montré par [26], que les PTH sont d'autant plus exposées à l'usure quand le pH est faible. En effet, les métaux ne sont pas neutres vis-à-vis des liquides avec lesquels ils sont en contact. Des phénomènes électrochimiques se produisent, à moins qu'il ne se forme rapidement, à la surface des pièces, une couche passive faisant écran. La stabilité de cette couche protectrice est fonction de nombreux paramètres : $\mathrm{pH}$, teneur en ions chlorures, pouvoir oxydant du milieu et contraintes d'origine mécanique. Si les matériaux employés en orthopédie sont à l'abri d'une corrosion généralisée, ils peuvent subir une corrosion localisée sous forme de corrosion par piqûres, corrosion caverneuse, corrosion soustension, fatigue-corrosion, frottement-corrosion, etc.

Dans notre expérimentation sur le rôle que peut jouer l'environnement sur l'usure de PTH, nous avons caractérisé le milieu acide par une solution Ringer de $\mathrm{pH}=$ 5,4 comparée à une solution normale de $\mathrm{pH}=5,7$.

Nous constatons qu'une diminution de $\mathrm{pH}$ de 0,3 correspond à une augmentation du taux de l'usure de $40 \mathrm{mg}$ au bout de 3,5 millions de cycles. Cette différence est d'autant plus significative $(P=0,0009)$ au fur et à mesure qu'on s'approche de la fin du stade 2 de l'usure (Fig. 14).

\subsection{Effet du procédé de stérilisation}

En général, la stérilisation des instruments chirurgicaux et du matériel d'ostéosynthèse en particulier prothèses et ancillaires, occupe une place de choix dans les milieux hospitaliers. La stérilisation des implants orthopédiques fait appel actuellement à trois méthodes différentes : autoclave, oxyde d'éthyle et irradiations par rayons gamma [27]. 


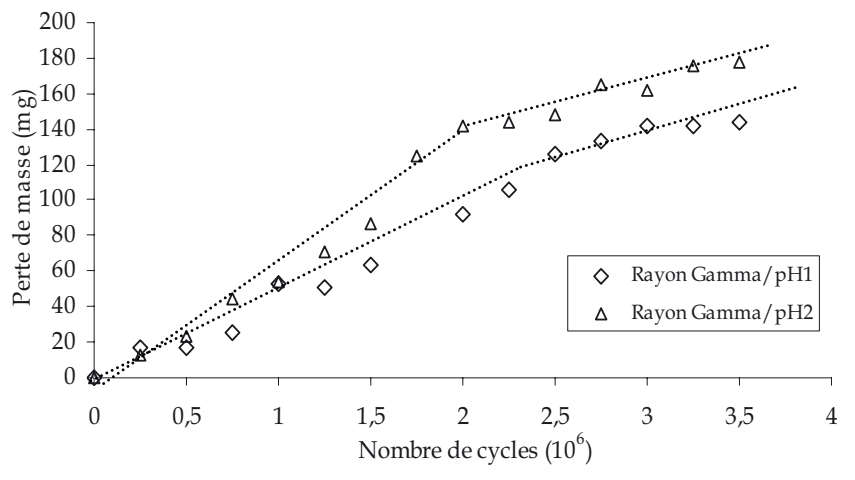

Fig. 14. Perte de masse sous l'effet du pH.

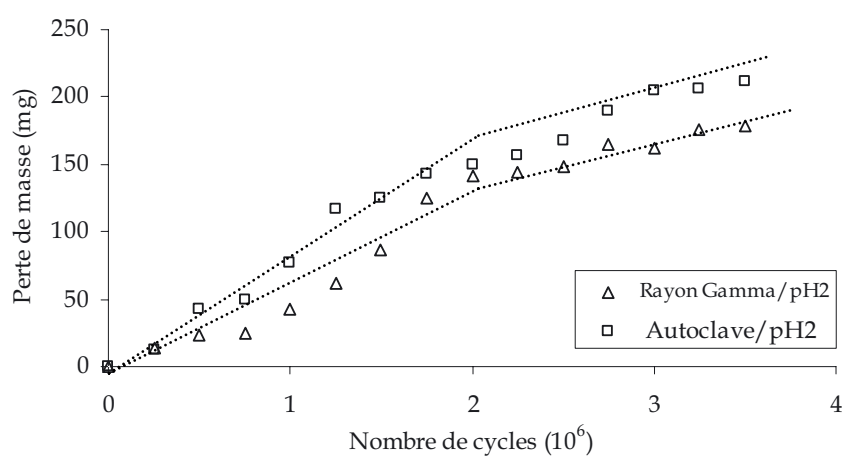

Fig. 15. Perte de masse sous l'effet du procédé de stérilisation.

Affatato et al. [28], montrent que le procédé de stérilisation des implants articulaires joue un rôle important dans la tenue à long terme des PTH. En comparant deux couples de matériaux testés à des conditions expérimentales semblables, et en utilisant deux procédés de stérilisation différents, ces auteurs [28-30] montrent que le polyéthylène stérilisé à l'oxyde d'éthyle (EtO) s'use plus rapidement que le polyéthylène stérilisé aux rayons gamma.

Selon les procédés de stérilisation des implants orthopédiques les plus utilisés à l'Institut M.T. Kassab, notre choix s'est fixé aux effets de la stérilisation par autoclave et irradiation gamma. Deux séries d'usure sur PTH de diamètre $28 \mathrm{~mm}$ sont faites sur le même couple de matériaux (UHMWPE/Inox 316L) : la première série de prothèses est stérilisée à l'autoclave et la seconde aux rayons gamma.

La perte de masse enregistrée sur le tribomètre pour les deux procédés de stérilisation montre la même tendance d'évolution en fonction du nombre de cycles (deux stades rapide et lent). Cependant, comparativement à la prothèse stérilisée à l'autoclave, on enregistre une réduction de la perte de masse sur la prothèse stérilisée aux rayons gamma, et une stabilisation de l'usure sur celle-ci pour une durée pouvant dépasser les $5 \times 10^{6}$ cycles. La prothèse stérilisée à l'autoclave subit une perte de masse plus rapide et à partir de 1,5 à 2 millions de cycles, le taux d'usure augmente, conduisant ainsi à une dégénérescence plus rapide des surfaces de contact entre tête et cotyle de PTH.

\section{Conclusion}

Cette étude expérimentale répond à un besoin clinique en matière de contrôle qualité des prothèses totales de hanche. Devant le coût élevé de l'analyse, la performance des PTH par l'étude clinique et les limites des essais usuels d'usure (pion/disque,...), les essais tribologiques sur simulateur offrent de meilleures possibilités.

Le taux de reprise des PTH observé dans beaucoup de centres orthopédiques justifie l'acquisition et l'introduction de ce matériel dans les laboratoires de recherche en biomécanique.

Les conclusions qui se dégagent de cette étude portent sur trois points.

La métrologie par analyse 3D peut être bénéfique pour étudier l'endommagement macroscopique des implants explantés (cotyle, tige de PTH,...). La collecte des informations peut aider à établir l'histoire du chargement imposé à la prothèse in vivo (direction de chargement,...).

La conception puis la réalisation et la validation d'un simulateur pour PTH reproduisant le milieu biologique (chargement + mobilité), ont permis d'apporter des éléments de réponse sur la tenue en service de plusieurs séries de prothèses type Müller, testées sous différentes conditions d'essais. Nous montrons qu'un milieu biologique caractérisé par un pH faible (plus acide), accélère l'usure de la prothèse. Une étude est programmée pour analyser l'effet d'antibiotiques sur la durée de vie des prothèses de hanche.

Le simulateur TUNIS-3DOF offre de plus la possibilité de programmer des sollicitations avec des pics de chargement pour simuler les chocs et les impacts sur la hanche prothésée.

\section{Références}

[1] R.M. Hall, A. Unsworth, B.M. Wroblewski, Wear in retreved Charnley acetabular sockets, ed. D. Dowson, Advances in medical tribology, London, Mechanical Engineering Publications Limited, 1988, pp. 57-67

[2] A. Fabre, Simulateur pour tester les prothèses de hanche à l'usure : modélisation et réalisation, Méc. Ind. Mat. 47 (1994) 370-373

[3] A. Boulila, Optimisation et aide à la conception d'implants orthopédiques, Étude de l'usure des implants articulaires de hanche, Thèse de doctorat en Mécanique, en cours, Rapport d'avancement septembre 2007, Tunis, $33 \mathrm{p}$.

[4] O. Calonius, Tribology of prosthetic joints, Validation of wear simulation methods, Acta polytechnica Scandinavica, Mech. Eng. Ser. 159 (2002) 62

[5] M. Skuteck, R.B. Bourne, S.J. MacDonald, International epidemiology of revision THP, Current Orthopaedics 20 (2006) 157-161

[6] F. Langlais, Le polyéthylène en 1997, Les Cahiers de la SOFCOT, Pathologies de la hanche de l'adulte, 1997, pp. $37-51$

[7] H.G. Willert, M. Semlitsch, Réactions de capsules articulaires à l'usure par frottement de matières plastiques et de métaux dans les endo-prothèses articulaires, Revue Technique SULZER 2 (1975) 119-133 
[8] M. Postel, M. Kerboull, J. Evrard, J.P. Courpied, Arthroplastie totale de hanche, ed. M. Postel, M. Kerboull, J. Evrard, J.P. Courpied, Berlin, Springer Verlag, 1985

[9] J.J. Caubert, Théorie et pratique industrielle du frottement, Collection mécanique des surfaces, DUNOD, 1962

[10] G. Zambelli, L. Vincent, Matériaux et contacts - une approche tribologique, 1998, Presses Polytechniques et universitaires de Romandes, ISBN 2-88074-338-9

[11] K.P. Judmann, G. Reinish, A. Pauschitz, F. Bauer, In vitro fatigue behaviour of a cemented acetabular reconstruction, J. Biomech. 39 (2006) 2882-2886

[12] P. Heaton Adegbile, N.P. Zant, J. Tong, Wear testing of total hip joints according to ISO and development of a new hip joint simulator according to ISO, J. Biomech. 39 (2006) 2882-2886

[13] S. Affatato, G. Bersaglia, I. Foltran, D. Emiliani, F. Traina, A. Toni, The influence of implant position on the wear of alumina-on-alumina studied in a hip simulator, Wear 256 (2004) 400-405

[14] A. Essner, G. Schmidig, A. Wang, The clinical relevance of hip joint simulator testing: in vitro and in vivo comparison, Wear 259 (2005) 882-886

[15] V. Saikko, T. Ahlroos, O. Calonius, Wear simulation of total hip prostheses with polyethylene against $\mathrm{CoCr}$, alumina and diamond-like carbon, Biomaterials 22 (2001) $1507-1514$

[16] Z.M. Jin, M. Stone, E. Ingham, J. Fisher, Biotribology, Current Orthopaedics 20 (2006) 32-40

[17] M. Douik, Apport de la biomécanique et des biomatériaux avancés pour la chirurgie orthopédique, point de vue des orthopédistes, $3^{\text {es }}$ Journées de biomécanique, biomatériaux avancés et Imagerie Médicale, 14-16 mai 2001, Tunis

[18] A.L. Galvin, S. Williams, P. Hatto, J. Thompson, G. Issac, M. Stone, E. Ingham, J. Fisher, Comparison of wear of ultra high molecular weight polyethylene acetabular cups against alumina ceramic and chromium nitride coated femoral heads, Wear 259 (2005) 972-976

[19] A. Boulila, K. Jendoubi, P. Chabrand, A. Zghal, Application de la méthode des plans d'expérience de Tagauchi dans la conduite des essais tribologiques sur prothèses totales de hanche, Mécanique \& Industries, en préparation
[20] J.G. Browsher, J.C. Shelton, A hip simulator study of influence of patient activity level on the wear of crosslinked polyethylene under smooth and roughened femoral conditions, Wear 250 (2001) 167-179

[21] J. Geringer, B. Forest, P. Combarde, Fretting - corrosion of materials used as orthopaedic implants, Wear 259 (2001) 943-951

[22] H.J. Cho, W.J. Wei,, H.C. Kao, C.K. Cheng, Wear behaviour of UHMWPE sliding on artificial hip arthroplasty materials, Materials Chem. Phys. 88 (2004) 9-16

[23] A. Essner, K. Sutton, A. Wang, Hip simulator wear comparison of metal-on metal, ceramic - on-ceramic and crosslinked UHMWPE bearings, Wear 259 (2005) 992-995

[24] S. Affatato, G. Bersaglia, M. Rocchi, P. Taddei, C. Fagmano, A. Toni Wear behaviour of crosslinked polyethylene assessed in vitro under severe conditions, Biomaterials 26 (2005) 3259-3267

[25] P. Kapsa, M. Cartier, Usure des contacts mécaniques, manifestations de l'usure, Techniques de l'ingénieur, Traité Génie Mécanique, BM 5 067, pp. 1-14

[26] R. Skalak, S. Chien, Handbook of bioengineering, ISBN 0-07-057783-8, McGraw, USA, 1987

[27] R. Kiok, S. Fernandes, S. Kupta, A. James, Sterilization methods of artificial joint prostheses, Group R2, April 23, 1997

[28] S. Affatato, G. Bersaglia, D. Emiliani, I. Foltran, P. Taddei, M. Reggiani, P. Ferrieri, A. Toni, The performance of gamma-and EtO-sterilised UHMWPE acetabular cups tested under severe simulator conditions, Part 1: role of the third-body wear process, Biomaterials 23 (2002) 4839-4846

[29] S. Affatato, G. Bersaglia, D. Emiliani, I. Foltran, P. Taddei, M. Reggiani, P. Ferrieri, A. Toni, The performance of gamma-and EtO-sterilised UHMWPE acetabular cups tested under severe simulator conditions, Part 2: wear particle characteristics with isolation protocols, Biomaterials 24 (2003) 4045-4055

[30] S. Affatato, B. Bordini, C. Fagnano, P. Taddei, A. Tinti, A. Toni, Effects of the sterilisation method on the wear of UHMWPE acetabular cups tested in a hip simulator, Biomaterials 23 (2002) 1439-1446 